2013

\title{
Pseudomorphic growth of InAs on misoriented GaAs for extending quantum cascade laser wavelength
}

Gregory Edward Triplett

Virginia Commonwealth University, getriplett@vcu.edu

Charles Meyer

Emily Cheng

See next page for additional authors

Follow this and additional works at: https://scholarscompass.vcu.edu/egre_pubs

Part of the Electronic Devices and Semiconductor Manufacturing Commons, and the Semiconductor and Optical Materials Commons

(C) 2013 American Vacuum Society

\section{Downloaded from}

https://scholarscompass.vcu.edu/egre_pubs/209

This Article is brought to you for free and open access by the Dept. of Electrical and Computer Engineering at VCU Scholars Compass. It has been accepted for inclusion in Electrical and Computer Engineering Publications by an authorized administrator of VCU Scholars Compass. For more information, please contact libcompass@vcu.edu. 
Authors

Gregory Edward Triplett, Charles Meyer, Emily Cheng, Justin Grayer, David Mueller, Denzil Roberts, and Samuel Graham 


\section{Pseudomorphic growth of InAs on misoriented GaAs for extending quantum cascade laser wavelength}

Charles Meyer, Emily Cheng, Justin Grayer, David Mueller, Gregory Triplett, Denzil Roberts, and Samuel Graham

Citation: Journal of Vacuum Science \& Technology A 31, 06F109 (2013); doi: 10.1116/1.4828357

View online: https://doi.org/10.1116/1.4828357

View Table of Contents: http://avs.scitation.org/toc/jva/31/6

Published by the American Vacuum Society

\section{Articles you may be interested in}

Orientation-dependent pseudomorphic growth of InAs for use in lattice-mismatched mid-infrared photonic structures

Journal of Vacuum Science \& Technology B, Nanotechnology and Microelectronics: Materials, Processing, Measurement, and Phenomena 32, 02C118 (2014); 10.1116/1.4867879

Buried heterostructure quantum cascade lasers with high continuous-wave wall plug efficiency Applied Physics Letters 91, 071101 (2007); 10.1063/1.2770768

$300 \mathrm{~K}$ operation of a GaAs-based quantum-cascade laser at $\lambda \approx 9 \mu \mathrm{m}$ Applied Physics Letters 78, 3529 (2001); 10.1063/1.1374520

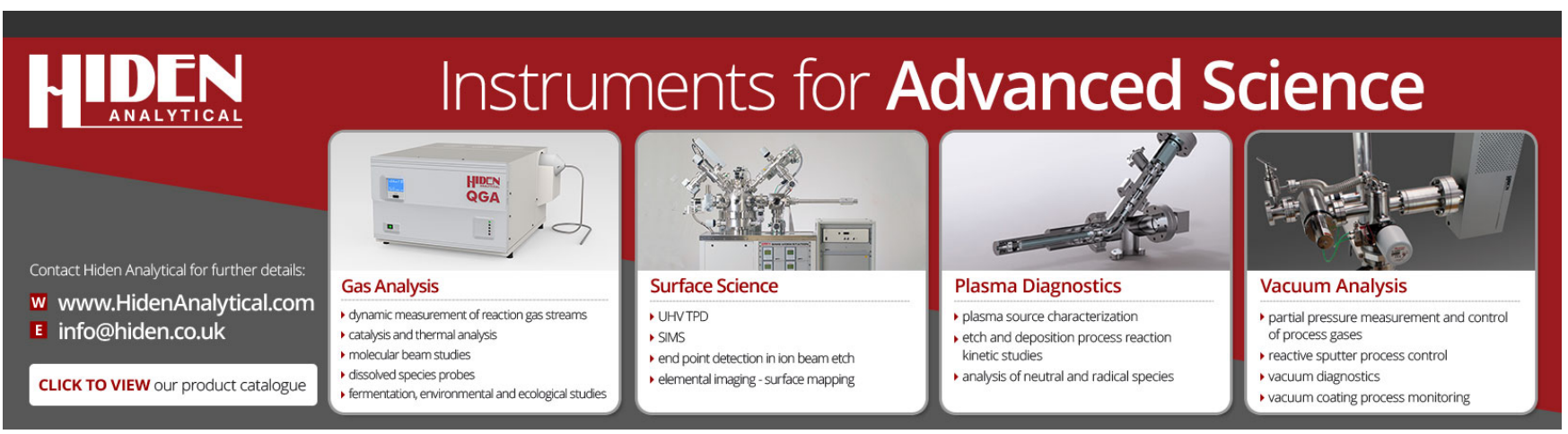




\title{
Pseudomorphic growth of InAs on misoriented GaAs for extending quantum cascade laser wavelength
}

\author{
Charles Meyer, Emily Cheng, Justin Grayer, David Mueller, Gregory Triplett, ${ }^{\text {a) }}$ \\ and Denzil Roberts \\ Department of Electrical and Computer Engineering, University of Missouri, 349 Engineering Building West, \\ Columbia, Missouri 65201 \\ Samuel Graham \\ Georgia Institute of Technology, Woodruff School of Mechanical Engineering, Atlanta, Georgia 30332
}

(Received 15 June 2013; accepted 17 October 2013; published 1 November 2013)

\begin{abstract}
The authors have studied the impact of epilayer strain on the deposition of InAs/GaAs on (100) and (111)B with $2^{\circ}$ offset toward $\langle 2-1-1\rangle$ surfaces. Consequences of a $7 \%$ lattice mismatch between these orientations in the form of three-dimensional growth are less apparent for (111)B with $2^{\circ}$ offset toward $\langle 2-1-1\rangle$ surfaces compared to (100). By exploring a range of molecular beam epitaxy process parameters for InAs/GaAs growth and utilizing scanning electron microscopy, atomic force microscopy, and Raman spectroscopy to evaluate the quality of these strained layers, the authors develop empirical models that describe the influence of the process conditions in regards to surface roughness with $>92 \%$ accuracy. The smoothest InAs/GaAs samples demonstrated average surface roughness of $0.08 \mathrm{~nm}$ for $10 \mu \mathrm{m}^{2}$ areas, albeit at very low deposition rates. The authors have found the most important process conditions to be substrate temperature and deposition rate, leading us to believe that controlling diffusion length may be the key to reducing defects in severely strained structures. InGaAs/AlGaAs quantum cascade laser structures were also produced on (111)B with $2^{\circ}$ offset toward $\langle 2-1-1\rangle$ to take advantage of the piezoelectric effect, and the modified laser transitions due to these effects were observed. (C) 2013 American Vacuum Society.
\end{abstract}

[http://dx.doi.org/10.1116/1.4828357]

\section{INTRODUCTION}

Indium arsenide (InAs) semiconductor devices that operate in the mid-infrared region are of interest, particularly for applications that include environmental monitoring, infrared countermeasures, and thermal imaging, as well as free space optics. ${ }^{1}$ These technologies are naturally leading the way toward highly integrated systems that include spectral sensitivity, temporal control, and spatial resolution. InAs remains an attractive semiconductor material for its inherent as well as modified properties (e.g., bandgap, carrier transport, and low deposition temperature) when incorporated with other materials. $^{2}$ The interests of this study are to explore epilayer formation and strain-induced piezoelectric effects in InAs/GaAs on (100) and (111)B with $2^{\circ}$ offset toward $\langle 2-1-1\rangle$ surfaces as a result of the difference in lattice constant between these two semiconductor materials $(\mathrm{GaAs}=5.65 \AA$ and $\mathrm{InAs}=6.06 \AA)$. Typically, InAs deposited on GaAs results in threedimensional features due to the compressive strain stemming from their large lattice mismatch $(\sim 7 \%){ }^{3,4}$ However, InAs growth on GaAs (111)B with $2^{\circ}$ offset toward $\langle 2-1-1\rangle$ has revealed the possibility of pseudomorphic, layer-by-layer formation, which opens up extraordinary possibilities to extend the performance of mid-infrared systems. ${ }^{5}$

The suppression of strain relief in lattice-mismatched structures is important for creating efficient, defect free photonic devices. In fact, taking advantage of InAs on GaAs (111)B with $2^{\circ}$ offset toward $\langle 2-1-1\rangle$ provides a unique

$\overline{{ }^{a} \text { Electronic mail: triplettg@missouri.edu }}$ opportunity to construct novel photonic emitters that utilize nonlinear optical processes, such as frequency mixing. ${ }^{6}$ Overcoming strain issues should lead to photonic devices with higher power in the mid-infrared region of the electromagnetic spectrum, thus meeting the demands for sensing and communications applications. ${ }^{7-9}$ The inherent electric fields in strained InAs/GaAs structures presents another degree of freedom for bandgap-engineered laser structures, e.g., quantum cascade lasers where the beam wavelength can be extended toward the near-infrared region. ${ }^{10}$ Figure 1 illustrates an example of a strained quantum cascade laser structure on (111) with incorporated InAs in the active region and the results of the induced piezoelectric effect.

The wavefunctions $\mathrm{E}_{1}, \mathrm{E}_{2}, \mathrm{E}_{3}$, and $\mathrm{E}_{4}$ highlight important laser transition states in this structure, where photons are produced through $\mathrm{E}_{4}-\mathrm{E}_{2}$ transitions and $\mathrm{LO}$ phonons through $\mathrm{E}_{2}-\mathrm{E}_{1}$ transitions. Whereas most quantum cascade lasers exhibit three wavefunctions $\left(E_{1}, E_{2}\right.$, and $\left.E_{3}\right)$, this design introduces a resonant $\mathrm{E}_{4}$ wavefunction because of the change in conduction band offset offered by indium incorporation into the GaAs matrix. The structure from left to right (with values in nanometers, n-doped underlined and active region in bold) is the following: $2.8,3.4,1.7,3.0,1.8,2.8,2.0,3.0$, 2.6, 3.0, 4.6, 1.9, 1.1, 5.4, 1.1, 4.8, 2.8, 3.4, 1.7, $3.0, \overline{1.8}, \overline{2.0}$, 3.0, 2.6, and 3.0. According to the Matthews and Blakeslee model, strained ternary (Ga)InAs layers on GaAs (100) can be synthesized up to at least 10 -nm with $25 \%$ indium composition. ${ }^{11}$ In this study, the goal is to explore the progression of deposition on (111)B with $2^{\circ}$ offset toward $\langle 2-1-1\rangle$ beyond this compositional range, primarily the binary InAs. 


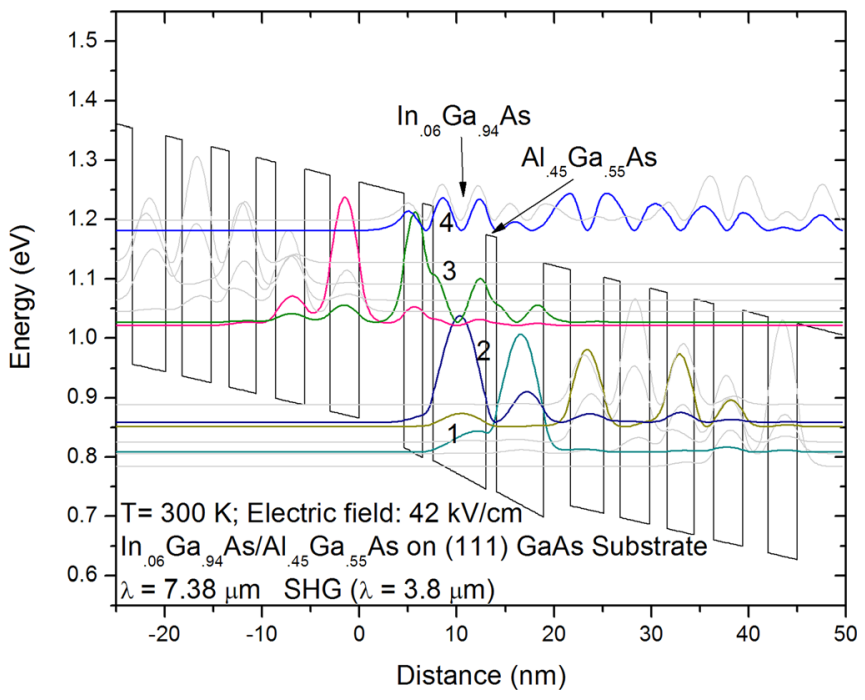

FIG. 1. (Color online) Simulated AlGaAs/InGaAs QCL bandstructure on (111) with a bias of $42 \mathrm{kV} / \mathrm{cm}$ and wavefunctions illustrating $\mathrm{E}_{4}-\mathrm{E}_{2}$ lasing and $\mathrm{E}_{2}-\mathrm{E}_{1}$ depopulation transitions.

Laser devices are routinely produced on various (100) orientation. Given that the deposition of laser structures is already complex, growth on (111) surfaces would involve additional strain and piezoelectric effects that should be considered in the initial device design. Figure 2 shows a simulation of an $\mathrm{AlGaAs} / \mathrm{GaAs}$ structure on a (111) surface, without indium in the active region quantum wells. On the other hand, deposition of $\mathrm{AlGaAs} /(\mathrm{In}) \mathrm{GaAs}$ on (111) surfaces introduces piezoelectric charges at the quantum well heterojunction as well as built-in electric fields in the quantum well. The promise of laser structures deposited on (111) surfaces lies in a tunable laser with a greater tuning range in which the gain peak of the laser cavity is manipulated. ${ }^{12}$

The piezoelectric effect stemming from strained superlattice growth on (111) surfaces is not a novel concept. ${ }^{13}$ There have been numerous studies on this topic as well as the actual magnitude of the piezoelectric field in bulk layers,

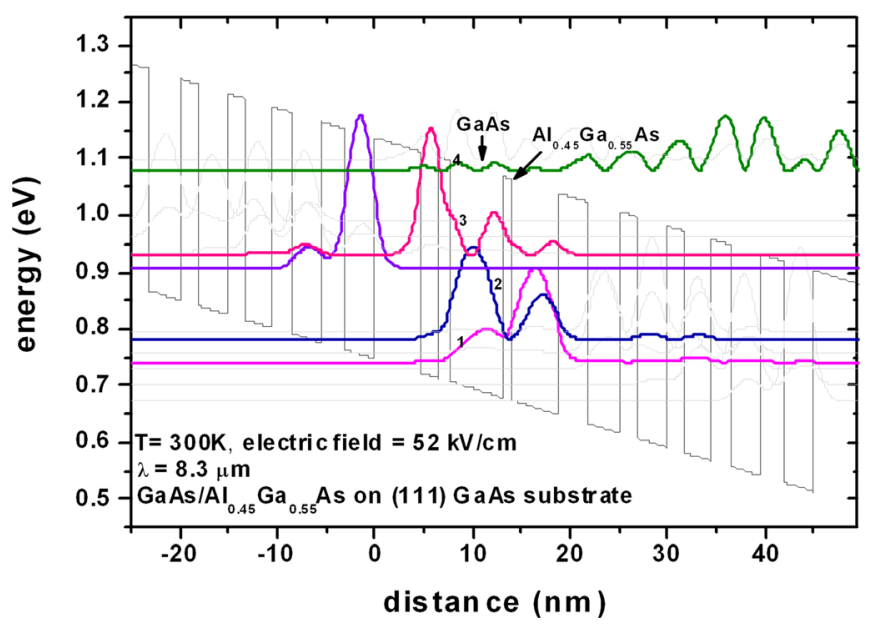

FIG. 2. (Color online) Simulated AlGaAs/GaAs QCL bandstructure on (111). The lack of indium in the active region means there are no piezoelectric charges at the quantum well heterojunction, hence no change in slope in the active region. quantum wells, ${ }^{14-16}$ and quantum dots. ${ }^{17,18}$ Still the interest in the optical and piezoelectric properties of films grown on (111) surfaces has been increasing. As an example, conventional laser devices on (111) surfaces have demonstrated notable advantages. ${ }^{12,19-22}$ Khoo et al. ${ }^{12}$ examined single quantum well $\mathrm{AlGaAs} / \mathrm{InGaAs}$ lasers grown on (111) GaAs substrates and measured low threshold current density as low as $87 \mathrm{~A} / \mathrm{cm}^{2}$. In another study by Deligeorgis et al., ${ }^{20}$ $\mathrm{AlGaAs} / \mathrm{InGaAs}$ laser diodes on (111) GaAs substrates were found to also have reduced threshold current compared to (100) devices. The sources of reduced threshold current were attributed to reduced density of states (especially in heavy-hole states) and spontaneous emission rate. Likewise, quantum cascade lasers on (111) surfaces has also been explored. In a study by Giovannini et al., ${ }^{21} \mathrm{AlInAs} / \mathrm{InGaAs}$ quantum cascade laser devices on InP were designed to emit at both $6 \mu \mathrm{m}$ and $10 \mu \mathrm{m}$ wavelength. Unlike laser diodes, reduced threshold currents were not observed. In fact, the threshold current was twice as large as the same structure on (100) surfaces. The sources of increased threshold current were attributed to one-order of magnitude higher doping in the (111) substrate and the optical quality of the structures. If the deposition process can be improved, a reduction in threshold current is expected and an improvement in power conversion efficiency can be obtained. This contributes to the motivation for this work.

The deposition of quantum cascade laser structures on (100) substrates has also been carefully studied. The deposition conditions are unlike those for (100) surfaces and the deposition window for (111) substrates is very narrow. ${ }^{23} \mathrm{In}$ essence, the material quality on (111) surfaces is very sensitive to the deposition conditions. Several groups have explored the various process ranges for arsenic-based material and found that good structural and electrical characteristics require lower arsenic overpressure along with higher growth temperature, which depends on the orientation angle of the (111) substrate. ${ }^{24,25}$ These conditions are contrary to the conditions necessary for growth on (100) surfaces. Cho et al. ${ }^{26}$ studied interfacial properties of strained piezoelectric InGaAs structures on (111) GaAs substrates. Results show that not only are atomically smoother interfaces possible for highly strained quantum wells, but also the longitudinal-optical phonon energy $(\sim 37 \mathrm{meV})$ is comparable with strained InGaAs wells grown on (100) surfaces. Therefore, the use of (In)GaAs in GaAs-based quantum cascade lasers should lead to promising devices. In another study by Marcadet $e t$ al., ${ }^{24} \mathrm{GaAs}$ step flow growth on (111) GaAs by molecular beam epitaxy was examined. In order to obtain mirrorlike surfaces, the governing molecular beam epitaxy parameters were substrate temperature and (arsenic to gallium) flux ratio. Results also show that surface morphology was driven by substrate orientation angle, where lower orientation $\left(0.5^{\circ}\right)$ has a higher growth temperature limit and higher orientation $\left(2^{\circ}\right)$ has a lower temperature limit. The difference in substrate temperature according to substrate orientation actually changes the Ga adatom surface diffusion length, which influences film morphology.

Substrate growth temperature (which controls the Ga adatoms surface diffusion length) is clearly responsible for 
smooth films. With stringent control of molecular beam epitaxy process parameters and understanding of the growth kinetics, excellent material quality for strained quantum cascade laser structures can be obtained. ${ }^{19}$ In this study, molecular beam epitaxy conditions are explored for strained InAs deposition on GaAs (111)B with $2^{\circ}$ offset toward $\langle 2-1-1\rangle$ surfaces that introduce a promising piezoelectric feature.

The key to extending device performance in these photonic structures is to address the limitations in the conduction band offset of $\mathrm{AlGaAs} / \mathrm{GaAs}$, while including resonant subbands in the active region. In this case, indium arsenide is alloyed with GaAs to increase the conduction band offset beyond that which is imposed by the AlGaAs alloy. Because of their non-centrosymmetric atomic arrangements, both GaAs and InGaAs are optically nonlinear. Therefore, the polarization (and thus the nonlinear susceptibility) of GaAs and InGaAs can be enhanced by inducing strain in these material layers. This is accomplished by increasing indium composition without degrading material in the form of structural defects.

To enhance nonlinear susceptibility, $\chi^{(2)}$, the aim is to maximize the product of the dipole matrix elements of the intersubband transitions and minimize the energy denominators using resonant effects, which can be approximated as follows: ${ }^{27}$

$$
\begin{aligned}
& \chi^{(2)}(2 \omega) \\
& \approx \frac{e^{3}}{\varepsilon_{0}} N \cdot \frac{\left\langle z_{i-i i}\right\rangle\left\langle z_{i i-i i i}\right\rangle\left\langle z_{i i i-i}\right\rangle}{\left(\hbar \omega-\Delta E_{i-i i}-i \cdot \Gamma_{i-i i}\right)\left(2 \hbar \omega-\Delta E_{i-i i i}-i \cdot \Gamma_{i-i i i}\right)},
\end{aligned}
$$

where $e$ is the electron charge, $\varepsilon_{0}$ the permittivity of the vacuum, $N$ is the electron density in the active region, $\Delta E_{i j}$ is the separation between subbands, $\Gamma_{i j}$ and $\left\langle z_{i j}\right\rangle$ are the half width at half maximum and the matrix element of the $i \rightarrow j$ intersubband transition. Nonlinear susceptibility can be optimized along with the tunability of oscillator strength in selectively strained laser cavities to achieve frequency mixing GaAs-based quantum cascade lasers. The role of the oscillator strength is critically important because it represents the likelihood of intersubband transitions from an upper state (i.e., $\mathrm{E}_{4}$ ) to a lower state (i.e., $\mathrm{E}_{2}$ ). In an effort to enhance frequency mixing, oscillator strength must be therefore optimized, where feasible. The oscillator strength (or optical transition strength) between electron states within quantum cascade structures can vary by taking into account their band nonparabolicity ${ }^{28}$ where the oscillator strength becomes

$$
f_{n^{\prime} n}=\frac{2 m_{o}}{\hbar^{2}}\left(E_{n^{\prime}}-E_{n}\right)\left|z_{n^{\prime} n}\right|^{2}
$$

with electron mass $m_{o}$, dipole matrix element $z_{n^{\prime} n}$, and the energy levels $E_{n^{\prime}}$ and $E_{n}$ of initial $n$ and final state $n^{\prime}$. Based on a diagonal transition, the matrix element can be tuned by applying an electric field normal to the layers. Enhancement of the $\left(\mathrm{E}_{4}-\mathrm{E}_{2}\right)$ optical transition can then be accomplished by increasing the electric field (due to the piezoelectric effect) within the device, which is available because of high-quality
TABLE I. MBE process parameter ranges.

\begin{tabular}{lcc}
\hline \hline Process parameters & Low & High \\
\hline Substrate temperature $\left({ }^{\circ} \mathrm{C}\right)$ & 480 & 500 \\
As:In beam equivalent pressure ratio & 10 & 42 \\
Deposition rate (Angstrom/s) & 0.0657 & 0.2818 \\
Deposition time (s) & 30 & 476 \\
Monolayers deposited & 0.65 & 17 \\
\hline \hline
\end{tabular}

(In)GaAs. By doing so, the $\left(\mathrm{E}_{4}-\mathrm{E}_{2}\right)$ optical transition becomes likely for photon generation (laser output).

\section{EXPERIMENT}

For each experiment, InAs structures were deposited simultaneously on (100) and (111)B with $2^{\circ}$ offset toward $\langle 2-1-1\rangle$ GaAs substrates in a solid-source molecular beam epitaxy reactor under a variety of process conditions (Table I). The reactor panels were cooled with flowing liquid nitrogen and maintained a base pressure on the order of $10^{-10}$ Torr. The (100) and (111)B with $2^{\circ}$ offset toward $\langle 2-1-1\rangle$ substrates were cleaved and indium-mounted side-by-side on 3 in. diameter molybdenum blocks and exposed to the same reactor conditions throughout the experiment. Reflection-high-energyelectron-diffraction (RHEED) analysis (using a 12-bit CCD camera) was employed on the (100) surface to confirm oxide desorption, determine deposition rate, and monitor deposition of the InAs epitaxial layers. After the native oxide was desorbed at approximately $580^{\circ} \mathrm{C}$, deposition of a $100-\mathrm{nm}$ GaAs buffer (smoothing) layer commenced before indium was deposited at the new substrate temperature. Atomic force microscopy data for the buffer layer is in Fig. 3. Sample rotation was employed except when determining deposition rates. Once samples were removed from the reactor, surfaces of each were analyzed using an FEI Quanta 600 FEG extended vacuum scanning electron microscope (SEM) and an Agilent atomic force microscope (AFM). The SEM was primarily used for large area analysis, while the AFM provided quantitative topography data for smaller regions. The surface roughness data were extracted using an image software package, and a full-factorial design of experiments and regression

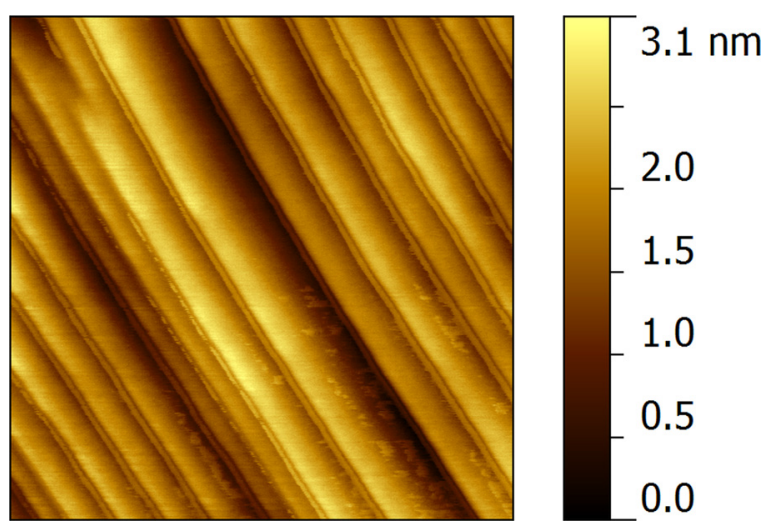

Fig. 3. (Color online) Atomic force microscopy scan of the GaAs buffer layer on misoriented (111)B GaAs with $2^{\circ}$ offset toward $\langle 2-1-1\rangle$ illustrating atomic step flow growth. 
algorithm were used to develop empirical models including beam equivalent pressure (BEP) ratio, substrate temperature, deposition rate, deposition time, and layer thickness as process inputs.

Using AFM data, InAs/GaAs samples were differentiated in terms of average roughness, root mean square roughness, average maximum roughness height, average third peak and third valley height, skewness, kurtosis, waviness average, root mean square waviness, average wavelength, and root mean square wavelength across $10 \mu \mathrm{m}^{2}$ area regions. Average roughness and RMS roughness are highlighted because they demonstrate the strongest correlation with the process parameters. The smoothest InAs/GaAs samples show surface roughness around $0.08 \mathrm{~nm}$, whereas the roughest surfaces have values around $0.7 \mathrm{~nm}$. As indicated in Figs. 4 and 5, AFM and SEM image comparison of (100) and (111)B with $2^{\circ}$ offset toward $\langle 2-1-1\rangle$ samples confirm that two-dimensional growth can be achieved on (111)B with $2^{\circ}$ offset toward $\langle 2-1-1\rangle$ surfaces without quantum dot formation that has been reported previously. ${ }^{29-31}$ The methods of strain compensation can be found elsewhere. ${ }^{31}$

\section{MODELING}

Regression models based on the molecular beam epitaxy process conditions and microscopy data are shown in Figs. 6 and 7. For simplicity, $r^{2}$ is presented to determine the measure of fit of each model. For average roughness (across

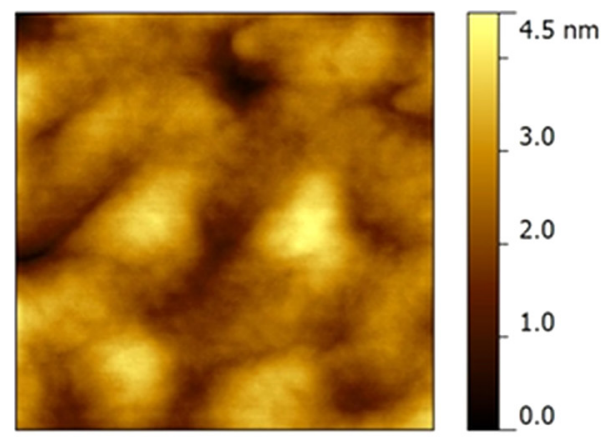

(A)
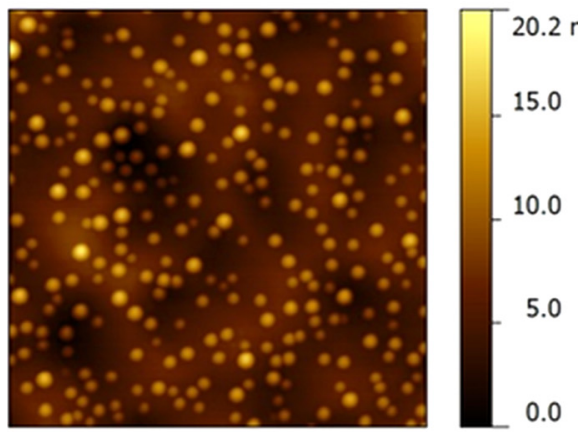

(C)
$10 \mu \mathrm{m}^{2}$ regions), the regression model has an $r^{2}$ value of 0.93 .The root mean square roughness model has a slightly better correlation with the data, with an $r^{2}$ value of 0.95 . In both cases, the most significant parameters were the temperature of the substrate and the deposition rate of InAs. Substrate temperature, beam equivalent pressure ratio, deposition time, and deposition rate are all significant, although the deposition rate and the substrate temperature have larger $t$-ratios, primarily because they have the greatest influence on diffusion length during InAs epitaxial growth.

\section{RESULTS AND DISCUSSION}

\section{A. Empirical model analysis}

Given that the surface reconstructions are starkly different between (111)B with $2^{\circ}$ offset toward $\langle 2-1-1\rangle$ and (100), we have observed that the range of process conditions for high quality deposition is relatively narrow. As a follow up, additional samples were produced where RHEED was observed on the (111)B with $2^{\circ}$ offset toward $\langle 2-1-1\rangle$ surfaces during deoxidation, rather than the (100). The only obvious difference was the $\sqrt{ } 19 \times \sqrt{ } 19 \mathrm{R} 23.4^{\circ}$ surface reconstruction pattern. Among all samples, the smoothest samples were produced with a very low deposition rate of $0.066 \AA / s$ and a substrate temperature of $480^{\circ} \mathrm{C}$. For the roughest surface, the substrate was also heated to $480^{\circ} \mathrm{C}$, but the growth rate was four-times faster than the smoothest sample. It appears

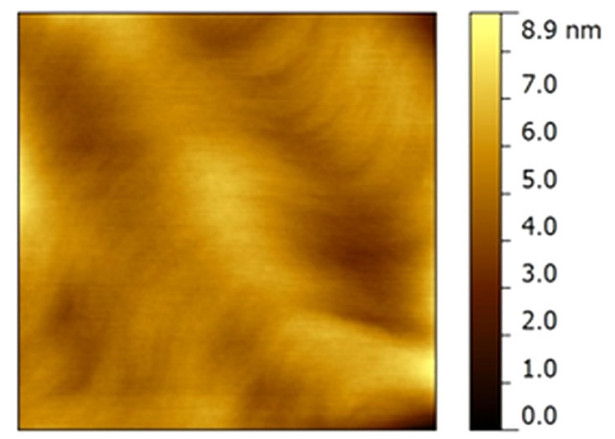

(B)

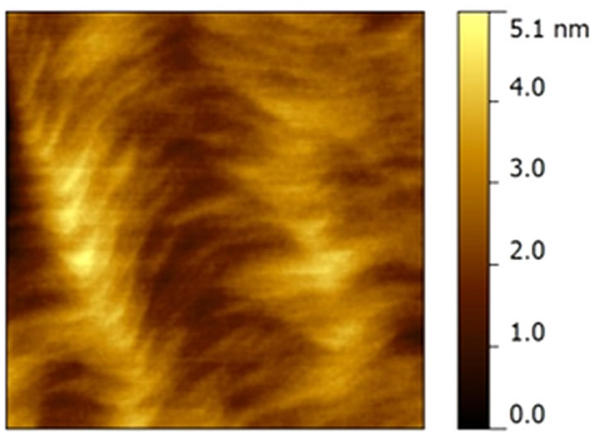

(D)

FIG. 4. (Color online) $1 \mu \mathrm{m} \times 1 \mu \mathrm{m}$ atomic force microscopy images of InAs/GaAs samples. (a) and (b) have 1.3 monolayers deposited at the same time at a substrate temperature of $480^{\circ} \mathrm{C}$, V:III BEP Ratio of $\sim 40: 1$, and a growth rate of. $024 \mu \mathrm{m} / \mathrm{h}$; (a) is on a (100) substrate and (b) is on a (111)B with $2^{\circ}$ offset toward $\langle 2-1-1\rangle$ substrate. (c) and (d) have 2 monolayers simultaneously deposited at a substrate temperature of $480{ }^{\circ} \mathrm{C}$, V:III BEP ratio of $\sim 40: 1$, and a growth rate of $0.024 \mu \mathrm{m} / \mathrm{h} ;(\mathrm{c})$ is on a (100) surface and (d) is on a (111)B with $2^{\circ}$ offset toward $\langle 2-1-1\rangle$ surface. The (100) surfaces all reveal three-dimensional growth, while the (111)B with $2^{\circ}$ offset toward $\langle 2-1-1\rangle$ surfaces show layer-by-layer growth. 


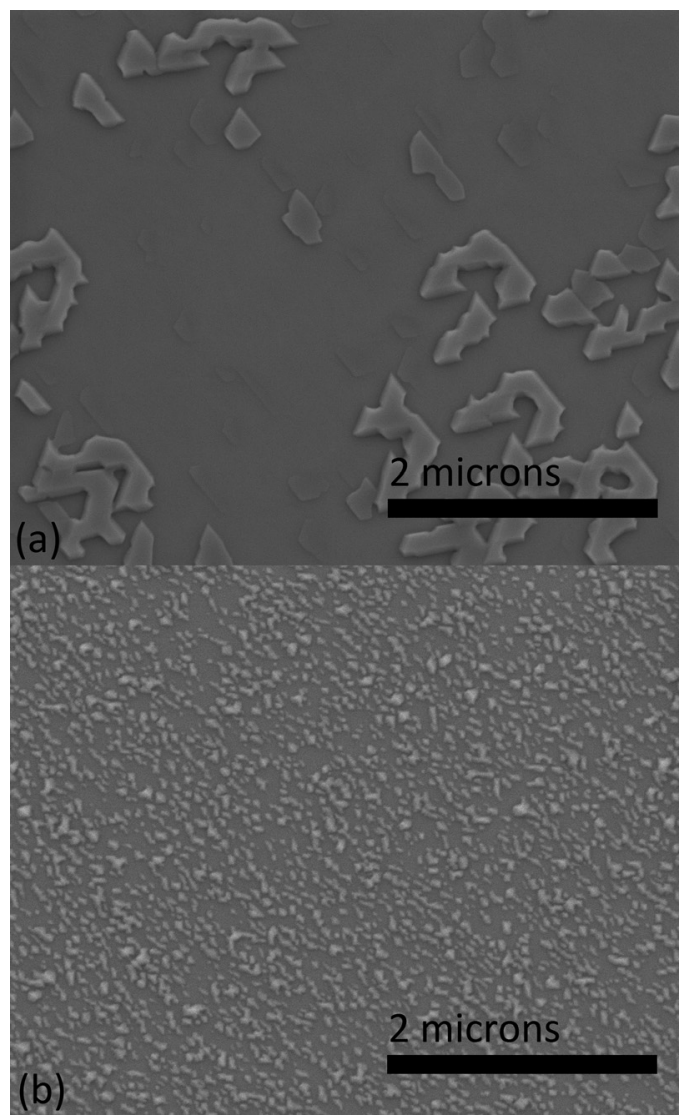

FIG. 5. Scanning electron microscopy images at 60000 magnification. (a) InAs on the misoriented (111) surface, demonstrating two-dimensional growth modes (b) InAs on (100) surface, revealing three-dimensional growth.

that cooler surface conditions (and therefore lower surface diffusion lengths) and slower deposition rate are more favorable for smooth, pseudomorphic epitaxial growth on misoriented (111)B.

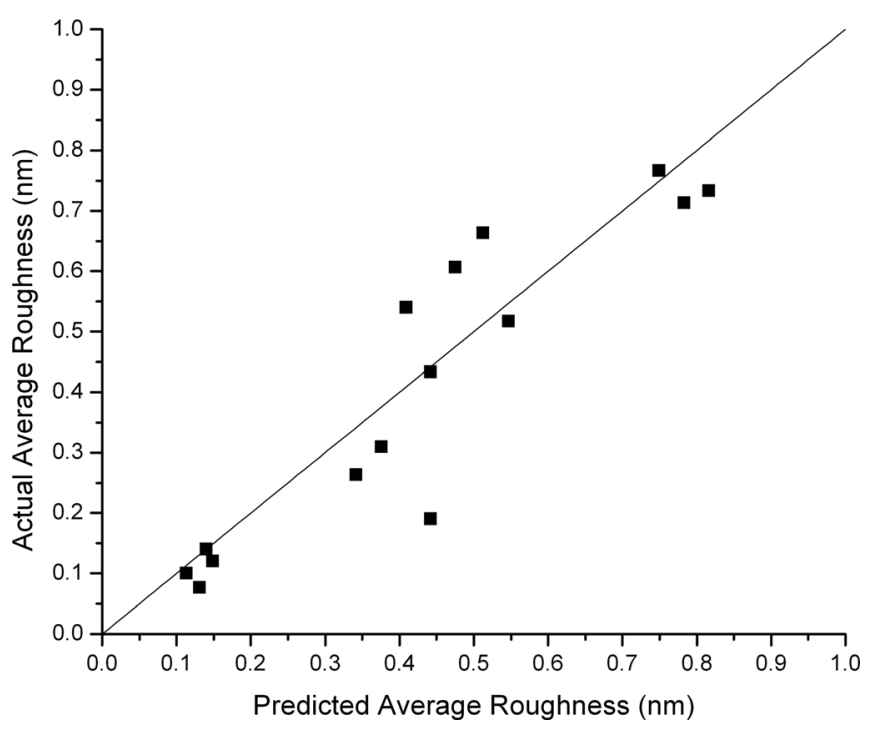

FIG. 6. Roughness average model fit for MBE-grown InAs/GaAs samples on (111)B with $2^{\circ}$ offset toward $\langle 2-1-1\rangle$ surfaces.

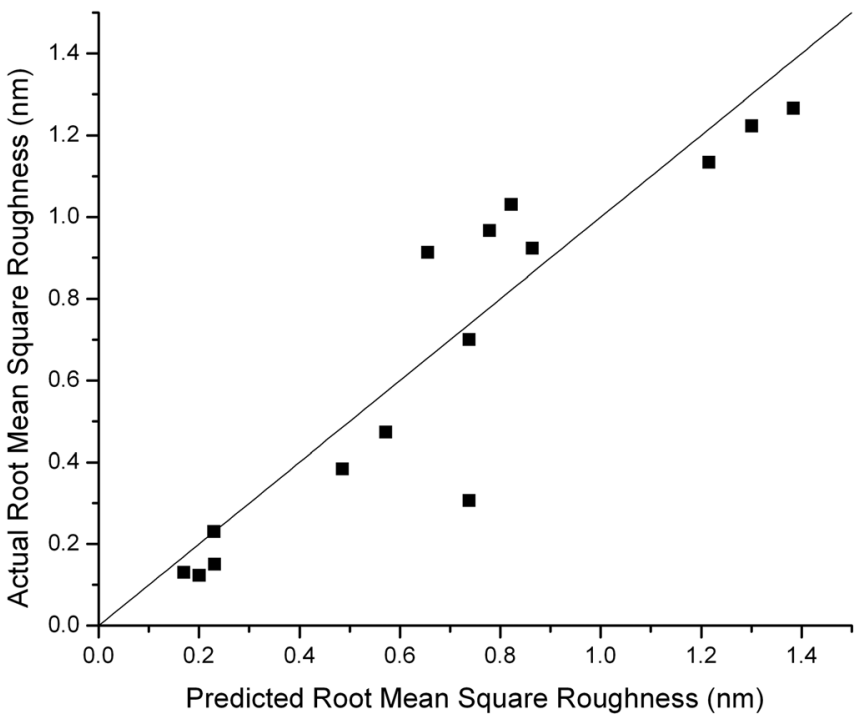

FIG. 7. Root-mean-square surface roughness model fit for MBE-grown InAs/GaAs samples on (111)B with $2^{\circ}$ offset toward $\langle 2-1-1\rangle$ surfaces.

\section{B. Quantum cascade laser Raman analysis}

InAs was also incorporated into the active region of GaAs/AlGaAs quantum cascade laser structures with the expectation that the piezoelectric effect would lead to frequency mixing $\left(\mathrm{E}_{4}-\mathrm{E}_{2}\right.$ rather than $\left.\mathrm{E}_{4}-\mathrm{E}_{3}\right)$. These samples, which contained more than 20 injector/active region pairs, were also analyzed using SEM, AFM, and Raman. Because four wavefunctions essentially determine the functionality of these laser structures, measuring wavefunction separations is necessary. Raman analysis in backscattering geometry was performed at room temperature using a $488 \mathrm{~nm}$ laser at $10 \mathrm{~mW}$ with a spot size diameter of approximately one micron. A notch filter was employed to remove the entire pump signal (near the $488 \mathrm{~nm}$ laser emission, $2.54 \mathrm{eV}$ ). Data were collected over $10 \mathrm{~s}$ intervals while averaging across ten individual scans. The expected Raman shifts are $33 \mathrm{meV}$ (due to GaAs), $37 \mathrm{meV}$ (due to AlAs), $7 \mu \mathrm{m}$ ( $\mathrm{E}_{3}-\mathrm{E}_{2}$ normal laser transition), and $3.8 \mu \mathrm{m}\left(\mathrm{E}_{4}-\mathrm{E}_{2}\right.$ transition-due to the frequency mixing) associated with the laser design. Figure 8 reveals all of the peaks mentioned above including the impact of (111)B with $2^{\circ}$ offset toward $\langle 2-1-1\rangle$ versus (100) surfaces (switching of intensity at 33 and 37), thus showing the piezoelectric effect has been utilized.

\section{SUMMARY AND CONCLUSIONS}

The deposition of InAs on GaAs (111)B $2^{\circ}$ offset toward $\langle 2-1-1\rangle$ has been analyzed using SEM, AFM, and Raman spectroscopy, and described using a surface roughness model. The InAs growth follows a two-dimensional, pseudomorphic growth pattern on (111)B with $2^{\circ}$ offset toward $\langle 2-1-1\rangle$, despite the $7 \%$ lattice mismatch. The roughness model reveals that substrate temperature and deposition rate significantly affect surface roughness. Based on these results, limitation of the diffusion length of adatoms during deposition via the substrate temperature and a low deposition rate appears to be the critical factors for creating smooth, defect free epitaxial InAs layers on GaAs (111)B $2^{\circ}$ offset 

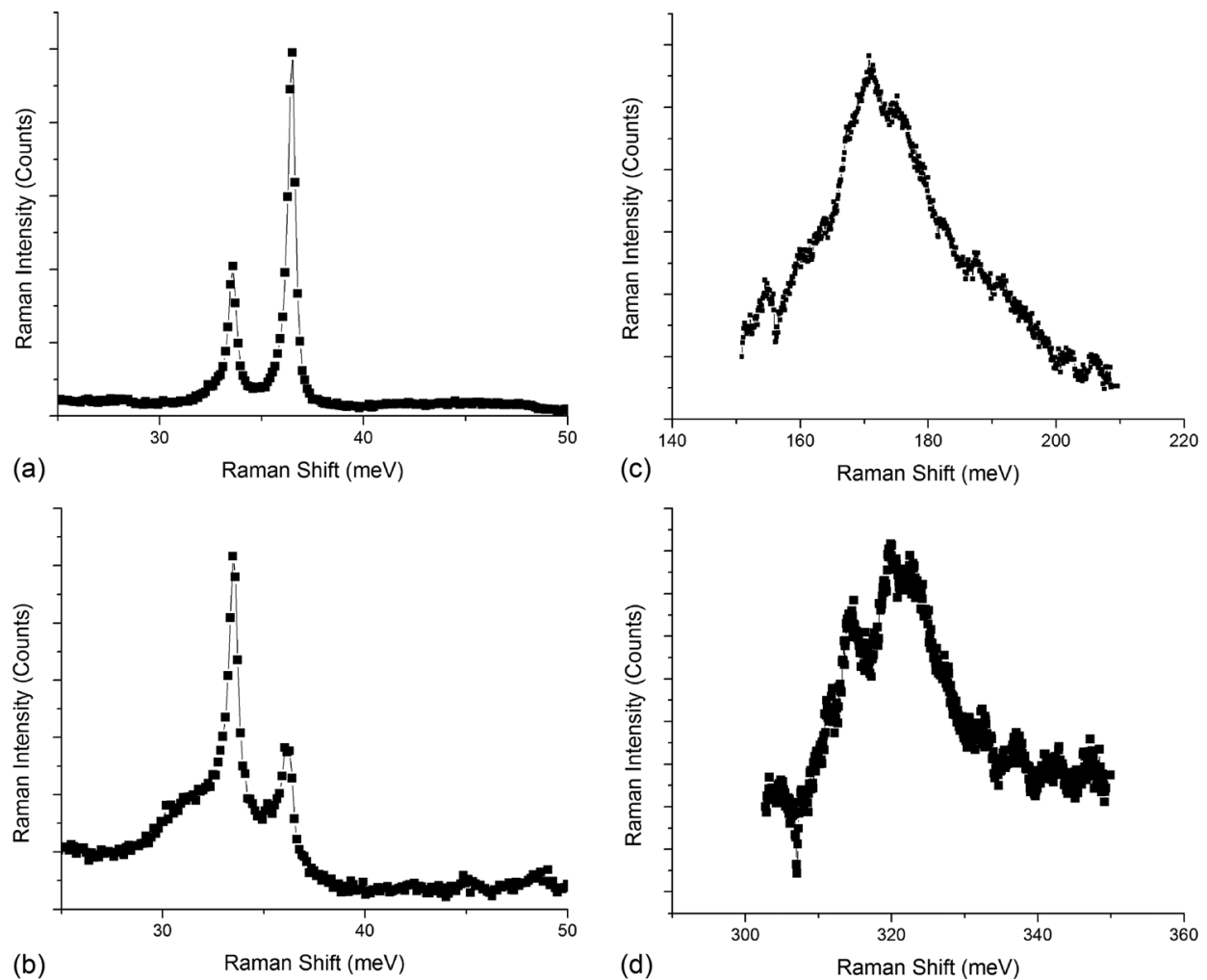

FIG. 8. Raman spectroscopy of a AlGaAs-(In)GaAs quantum cascade laser structures illustrating (a) the Raman shift due to absorption on GaAs (100) surfaces, (b) the Raman shift due to absorption on GaAs (111)B $2^{\circ}$ offset toward $\langle 2-1-1\rangle$, (c) the $\mathrm{E}_{3}-\mathrm{E}_{2}$ intersubband transition, and (d) the $\mathrm{E}_{4}-\mathrm{E}_{2}$ intersubband transition, suggesting frequency mixing within the active region, in accordance with the laser design.

toward $\langle 2-1-1\rangle$. Smooth epitaxial growth, coupled with the compressive strain from the large lattice mismatch of InAs and its alloy with GaAs, have been utilized to create a novel quantum cascade laser device that produces photonics in the near infrared region for advanced communications and sensing applications. Utilization of the piezoelectric effect in multiquantum well structures introduces a new dimension in photonic device design.

\section{ACKNOWLEDGMENT}

This work was supported by AFOSR Young Investigator Program Grant Number FA9550-10-1-0482.

${ }^{1}$ F. Capasso et al., IEEE J. Quantum Electron. 38, 511 (2002).

${ }^{2}$ S. E. Hooper, Semicond. Sci. Technol. 8, 1069 (1993).

${ }^{3}$ L. Goldstein, F. Glas, J. Marzin, M. Charasse, and G. Le Roux, Appl. Phys. Lett. 47, 1099 (1985).

${ }^{4}$ M. Grundmann, O. Stier, and D. Bimberg, Phys. Rev. B 52, 11969 (1995).

${ }^{5}$ D. Mueller, D. Roberts, and G. Triplett, J. Electron. Mater. 41, 959 (2012).

${ }^{6}$ G. Triplett and D. Roberts, IEEE J. Quantum Electron. 46, 1782 (2010).

${ }^{7}$ F. K. Tittel, D. Richter, and A. Fried, in Solid-State Mid-Infrared Laser Sources (Springer, Berlin, 2003), pp. 458-529.

${ }^{8}$ P. Werle, F. Slemr, K. Maurer, R. Kormann, R. Mücke, and B. Jänker, Opt. Laser. Eng. 37, 101 (2002).

${ }^{9}$ C. H. Chan, M. C. Chen, H. H. Lin, Y. F. Chen, G. J. Jan, and Y. H. Chen, Appl. Phys. Lett. 72, 1208 (1998).

${ }^{10}$ B. Laurich, K. Elcess, C. Fonstad, J. Beery, C. Mailhiot, and D. Smith, Phys. Rev. Lett. 62, 649 (1989).

${ }^{11}$ J. W. Matthews and A. E. Blakeslee, J. Cryst. Growth 27, 118 (1974).

${ }^{12}$ E. A. Khoo, A. S. Pabla, J. Woodhead, J. P. R. David, R. Grey, and G. J. Rees, Electron. Lett. 33, 957 (1997).

${ }^{13}$ C. Mailhiot and D. L. Smith, Phys. Rev. B: Condens. Matter 37, 10415 (1988).
${ }^{14}$ K. W. Goossen, E. A. Caridi, T. Y. Chang, J. B. Stark, D. A. B. Miller, and R. A. Morgan, Appl. Phys. Lett. 56, 715 (1990).

${ }^{15}$ T. B. Bahder, Phys. Rev. B: Condens. Matter 51, 10892 (1995).

${ }^{16}$ T. S. Moise, L. J. Guido, and R. C. Barker, J. Appl. Phys. 74, 4681 (1993).

${ }^{17}$ G. Bester and A. Zunger, Phys. Rev. B: Condens. Matter 71, 45318 (2005).

${ }^{18}$ V. Ranjan, G. Allan, C. Priester, and C. Delerue, Phys. Rev. B: Condens. Matter 68, 115305 (2003).

${ }^{19}$ X. Marcadet, V. Ortiz, J. Y. Bengloan, S. Dhillon, M. Calligaro, and C. Sirtori, J. Vac. Sci. Technol. B 22, 1558 (2004).

${ }^{20}$ G. Deligeorgis, G. E. Dialynas, Z. Hatzopoulos, and N. T. Pelekanos, J. Phys.: Conf. Ser. 10, 35 (2005).

${ }^{21}$ M. Giovannini, M. Beck, N. Hoyler, and J. Faist, J. Appl. Phys. 101, 103107 (2007).

${ }^{22}$ G. E. Dialynas, G. Deligeorgis, N. Le Thomas, Z. Hatzopoulos, and N. T. Pelekanos, presented at the Fifth International Workshop on Epitaxial Semiconductors on Patterned Substrates and Novel Index Surfaces (ESPSNIS), Stuttgart, Germany, 13-15 October 2003 (unpublished).

${ }^{23}$ W. Yeo, R. Dimitrov, W. J. Schaff, and L. F. Eastman, Appl. Phys. Lett. 77,2764 (2000).

${ }^{24} \mathrm{X}$. Marcadet, J. Olivier and J. Nagle, presented at the Sixth International Conference on the Formation of Semiconductor Interfaces, Cardiff, UK, British Assoc. Crystal Growth. British Tourist Authority, IOP, 23-27 June 1997 (unpublished).

${ }^{25}$ T. Hayakawa, M. Morishima, and S. Chen, Appl. Phys. Lett. 59, 3321 (1991).

${ }^{26}$ C. Soohaeng, A. Sanz-Hervas, K. Jongseok, A. Majerfeld, and B. W. Kim, J. Appl. Phys. 96, 1909 (2004).

${ }^{27}$ F. Capasso, C. Sirtori, and A. Y. Cho, IEEE J. Quantum Electron. 30, 1313 (1994).

${ }^{28}$ C. Sirtori, F. Capasso, J. Faist, and S. Scandolo, Phys. Rev. B: Condens. Matter 50, 8663 (1994).

${ }^{29}$ H. Yamaguchi, M. Fahy, and B. Joyce, Appl. Phys. Lett. 69, 776 (1996).

${ }^{30}$ L. A. Zepeda-Ruiz, D. Maroudas, and W. H. Weinberg, Surf. Sci. 418, L68 (1998).

${ }^{31}$ H. Wen, Z. M. Wang, J. L. Shultz, B. L. Liang, and G. J. Salamo, Phys. Rev. B 70, 205307 (2004) 